\title{
Large-Scale, Preparative Paper Chromatography
}

\author{
Harriet L. Frush \\ Institute for Materials Research, National Bureau of Standards, Washington, D.C. 20234
}

(September 8, 1966)

\begin{abstract}
Simplified techniques are described for the use of heavy papers in large-scale, preparative chromatography. A new technique is illustrated for nondestructively locating bands of compounds on wet chromatograms. Capacities and behavior characteristics of Whatman seed-test paper and Whatman No. 17 paper are illustrated.
\end{abstract}

Key Words: Chromatography on seed-test paper, large-scale paper chromatography, radioactive carbohydrates, use of heavy papers in preparative chromatography.

\section{Introduction}

Most of the techniques that have been described for large-scale, preparative paper chromatography depend on an increase in bulk through the use of many closely pressed layers of a thin paper [1]. ${ }^{1}$ Some of these methods require the tedious loading of each sheet, and others tend to suffer from a lack of uniformity in the movement of compounds throughout the bulk. The latter disadvantage has been overcome in one commercial apparatus by empirically regulating the pressure [2].

In 1957, Brownell and co-workers [3] described a large-scale, heavy-paper technique for preparative chromatography in which, after a preliminary treatment, the paper is used in essentially the customary manner. The authors stated that the dense paper (Whatman seed-test paper, $1.5 \mathrm{~mm}$ thick) is capable of separating 0.5 to $3.0 \mathrm{~g}$ of material per large sheet (18 $\times 22$ in), depending on the spread in $R_{f}$ values. $^{2}$ They also described a method for nondestructively locating the separated bands by staining a "print" obtained by pressing a sheet of thin paper against the wet chromatogram.

However, despite the unusually high capacity of the heavy paper, and the inherent advantages of paper chromatography over column chromatography, the techniques described by Brownell et al. have not been widely adopted, presumably because of several difficulties encountered in using the heavy paper. Thus, (a) the edge of the paper must be provided with a machine-stitched stirrup or loop (for supporting the

' Figures in brackets indicate the literature references at the end of this paper.

2 The use of this paper for chromatography had been suggested earlier [4]. paper on a rod) and with a stitched wick of a thinner paper. $^{3}$ (b) Because the seed-test paper is not pure cellulose, it must be chromatographically washed with dilute acid and with water; this treatment requires several days. Finally, (c) the movement of the bands through the paper is not as uniform as that in papers made of pure cellulose.

Despite these disadvantages, heavy-paper chromatography has found extensive application in this laboratory in the large-scale separation of mixtures of carbohydrates and related compounds, both radioactive and nonradioactive. Certain simplified techniques, described below, have facilitated application of the paper to problems of separation and may prove useful in other laboratories. Chief among these techniques is a more precise method for nondestructively locating the bands of compounds on the developed chromatograms.

The techniques described are equally applicable to Whatman No. 17 paper, and, on this paper, afford satisfactory separations of 0.2 to $0.6 \mathrm{~g}$ per large sheet. The No. 17, like the seed-test paper, is a "fast" paper whose properties are also improved by use of a thinner wick.

\section{Preparation of the Heavy Paper}

A strip of Whatman No. $3 \mathrm{MM}$ paper $(7 \times 18$ in) is loosely machine-stitched with white, mercerized, heavy-duty thread along each short side, about 2 in

\footnotetext{
${ }^{3}$ The use of a wick of a thinner paper to retard the movement of a solvent through a "fast," thick paper had been described [5]. Slower movement of a solvent affords more complete equilibration and sharper separation.

A patent [6] describes the use of a seed-test paper, without a wick, for rapid, large-scale purifications; for example, 100 sheets $(3 \mathrm{~mm}$ thick) were used in purifying $220 \mathrm{~g}$ of aniline in $6 \mathrm{hr}$. It appears doubtful that compounds having similar $R_{f}$ values can be separated by such rapid chromatography.
} 
from the edge. The strip is then folded as shown in figure 1 , to form both a stirrup and a wick. The 18-in edge of the seed-test paper is softened by momentarily dipping it in water, and the excess is thoroughly blotted. The dampened edge is inserted about $3 / 4$-in into the folded strip, and the two papers are twice machinestitched together. During washing of the paper (and, occasionally, during development of a chromatogram), it is desirable to use an auxiliary wick to facilitate flow of solvent. However, the second wick need not be sewn, but may be added as shown in figure 2 . The additional wick is inserted near the base of the stirrup, and functions as well as if it had been sewn in place. To facilitate dripping of the solvent, the lower edge of the heavy paper is conveniently serrated by dampening it as described above and tearing off small pieces of the paper so as to form an irregular edge.

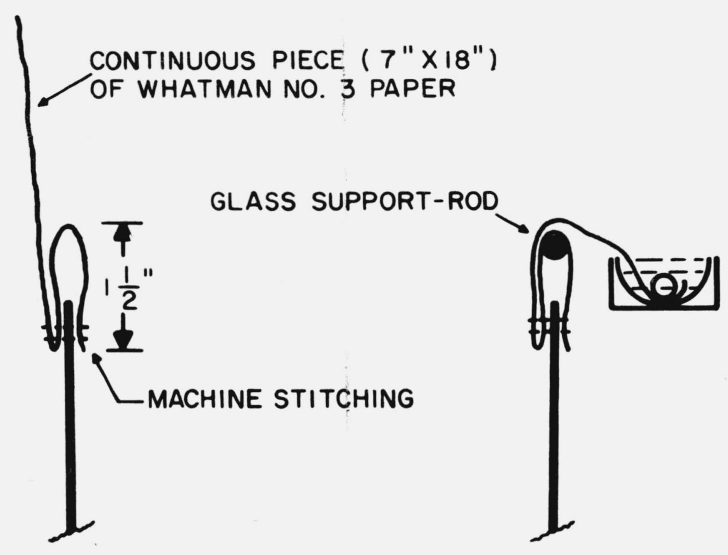

FIGURE 1. Preparation of stirrup and wick (cross section).

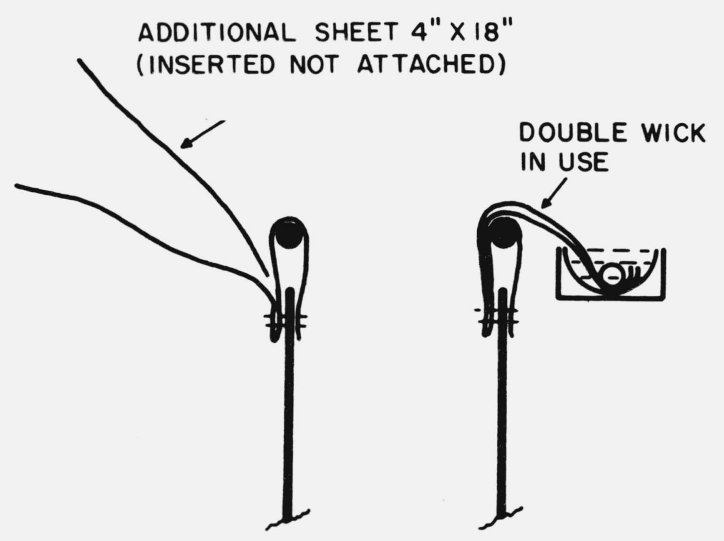

Papers are usually prepared in groups of eight, and simultaneously washed in a commercial chromatography cabinet having four troughs, each of which holds the wicks from two papers. Four external, 500-ml, leveling bulbs supply the solvent through four holes in the cabinet lid, each hole being situated above the middle of a trough. The papers are washed, with $0.01 N$ hydrochloric acid and then with water, as described by Brownell et al., except that the total time of washing is reduced from seven to four days (two for each solvent). The sheets are air-dried, and strips ( 1 in or more in width) are cut from each long edge that appears to be thin; the stirrups and wicks are trimmed to match. If the papers are used within two or three weeks after preparation, no further washing is necessary. If they are kept for longer periods, an additional washing is given, before use, with the solvent to be employed in elution of the bands (frequently, $50 \%$ aqueous methanol). This washing removes some color that develops in the paper on standing.

Before application of the sample, a narrow band $(<0.5 \mathrm{~cm}$ wide $)$ is drawn with a pencil about $2 \mathrm{~cm}$ below the stirrup and on both sides of the paper. Aqueous or alcoholic solutions of mixtures (as much as $2 \mathrm{ml}$ ) are applied to this band by means of a commercial loading pipet having a $1-\mathrm{ml}$ bulb and a short tip of capillary tubing. The pipet affords excellent control of the solution, which flows only on contact of the tip with the paper. The band is loaded from both sides of the paper, which is then air-dried for several hours.

Development with the solvent of choice is carried out in the chromatography cabinet with the aid of the leveling bulbs, and the use of either one or two wicks per sheet. Two wicks are employed with a slow-moving solvent, such as one containing tertpentyl alcohol; only one wick is necessary with less viscous solvents, such as ethyl acetate or 2-butanone.

Preparation of the No. 17 or similar paper is simpler. The same stirrup and wick are stitched to the paper (which need not be previously moistened), but the addition of the auxiliary wick is unnecessary. The paper is used without prior purification or trimming.

\section{Nondestructive Location of Bands on the Developed Chromatogram}

When development is judged to be satisfactory, the wet chromatogram is laid on a glass plate in a well ventilated hood, and is overlaid with a sheet of thin paper (such as Whatman No. 1) carefully positioned at the origin. A small, plastic whee ${ }^{4}$ is then drawn down the paper in a series of straight lines (see fig. 3). By pressing the thin paper into the wet chromatogram, the wheel causes good (and fairly uniform) absorption of the solvent in narrow, vertical lines (about $3 \mathrm{~mm}$ wide). The chromatogram is temporarily returned to the cabinet, and the tracing may be sprayed with a suitable color reagent. Because the color is intense

FIGURE 2. Addition of auxiliary wick in order to increase flow of solvent. 
THIN PAPER LAID OVER THICK WET CHROMATOGRAM

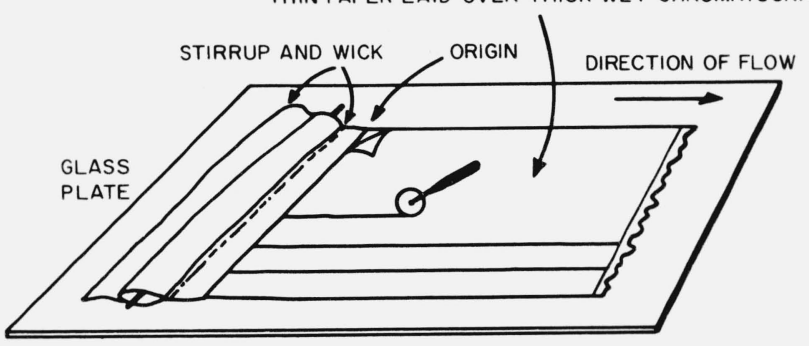

Figure 3. Preparation of tracing.

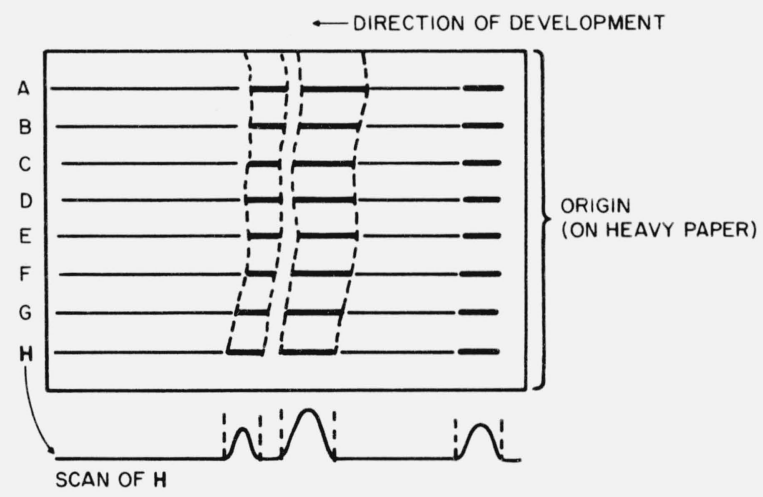

FIGURE 4. Use of tracing in chromatography of tritiated compounds.

in the lines of absorption, limits of the bands are more clearly defined than in a "print" [3] of the entire chromatogram. Spraying of alternate strips, or of two different tracings, with different color reagents sometimes gives additional information concerning the compounds. (If it is desired only to ascertain the progress of a development approximately, a single line, drawn by the wheel on a strip of thin paper placed lengthwise on the middle of the wet chromatogram, will suffice. $)^{5}$ When the separation of bands has become satisfactory, and a tracing has clearly indicated their limits, the chromatogram is air-dried.

For compounds labeled with carbon-14, a radioautograph is the most accurate means of locating bands on a chromatogram. However, the progress of the completeness of separation may be quickly ascertained by use, on a strip of thin paper, of the tracing technique described above. The strip is then scanned for radioactivity in a paper-chromatogram scanner having a $1: 1$ correspondence of chromatogram and chart.

For tritium-labeled compounds, the tracing technique has a further advantage. Tritium does not produce a good radioautograph of a chromatogram, because of the weakness of its radiation. If the compounds are not present in sufficient quantity to be clearly defined on the tracing by means of a color reagent, the technique illustrated in figure 4 is employed. In this, the individual lines produced by the plastic wheel are scanned for radioactivity, and the positions recorded for the peaks are used to locate the bands. This technique has been employed in the separation of high-activity tritium-labeled compounds on the lower capacity papers (such as Whatman No. $3 \mathrm{MM}$ ).

\section{Removal and Treatment of Bands of the Developed Chromatogram}

Bands sketched on a tracing may be outlined on the heavy chromatogram merely by positioning the tracing and using a sharp pencil or stylus. Band outlines from a radioautograph are conveniently transferred to the chromatogram by (a) puncturing the film to form series of small holes outlining the bands, (b) positioning the film on the chromatogram, ${ }^{6}$ and (c) dusting the film with charcoal. The series of black dots produced on the chromatogram adequately and accurately outline the bands, and the intact film may be saved for reference. Seed-test paper is extremely difficult to cut with scissors, and it tends to crumble when cut with a sharp knife. However, the bands are conveniently removed by means of tinners' shears.

For elution of a band, the stirrup and wick are attached to one narrow edge, not by sewing, but by use of a series of vertically-placed, stainless-steel staples. The auxiliary wick is added as shown in figure 2 , and the band, trimmed to a point at the lower end, is eluted in the chromatography cabinet, usually with 50 percent aqueous methanol. Subsequent treatment of the eluant usually includes the use of small amounts of a decolorizing carbon, and, in some instances, of ionexchange resins.

\section{Capacity of the Heavy Papers}

Figures 5 and 6 show some results of a study made of the capacity of the seed-test and the No. 17 paper, respectively. Obviously, the ease of separation depends on the spread in $R_{f}$ values and on the quantities separated. At most indicated levels of loading, L-rhamnose was satisfactorily separated from both D-glucose and D-fructose. Separation of the last two from each other by further development or by a second development is feasible on the seed-test paper at the l-g level, and on the No. 17 paper at the 400-mg level. It is possible to increase the capacities of the heavy papers somewhat (if the decrease in length is unimportant) by attaching the stirrup and wick to the
${ }^{5}$ In a recently described technique for ascertaining the progress of a development [7], the chromatogram is dried, and a strip of pressure-sensitive tape is applied lengthwise, peeled off with the adhering fibers, and sprayed with a color reagent. The technique described in the present paper permits monitoring of the wet chromatogram. If the separation of bands is insufficient, the development can be continued immediately.
${ }^{6}$ In this laboratory, film is stapled to the chromatogram for the preparation of a radioautograph, and the puncture marks from the staples subsequently afford a convenient means for precisely positioning the developed radioautograph on the chromatogram. 


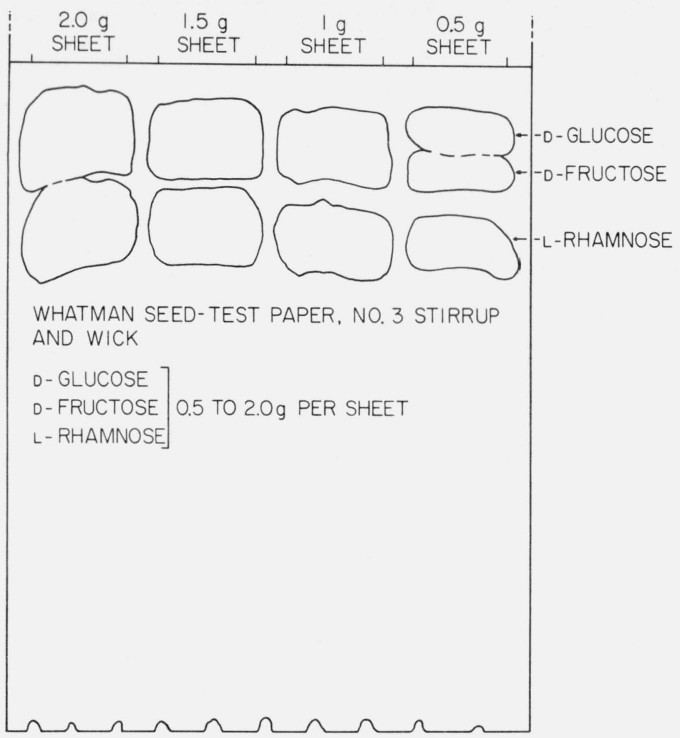

FIGURE 5. Illustration of capacity of seed-test paper (solvent: 1-butanol-acetic acid-water 4:1:5, upper layer).

long (22-in) edge of the paper. In the two directions, the chromatographic properties of the seed-test paper are the same. The $R_{f}$ values for the seed-test paper are about 0.7 of those for Whatman paper No. 1 .

\section{Summary}

Simplified techniques are described for the use of heavy papers in large-scale, preparative chromatography. Bands of compounds on the developed chromatogram are nondestructively located by laying the wet chromatogram on glass, overlaying it with a thin paper, and pressing a series of lines down the length of the upper paper by means of a small, plastic wheel. When this tracing is stained, limits of the bands are clearly defined. Capacities and behavior characteristics of Whatman seed-test paper and Whatman No. 17 paper are illustrated. The $R_{f}$ values for Whatman seed-test paper are about 0.7 of those for Whatman paper No. 1 .

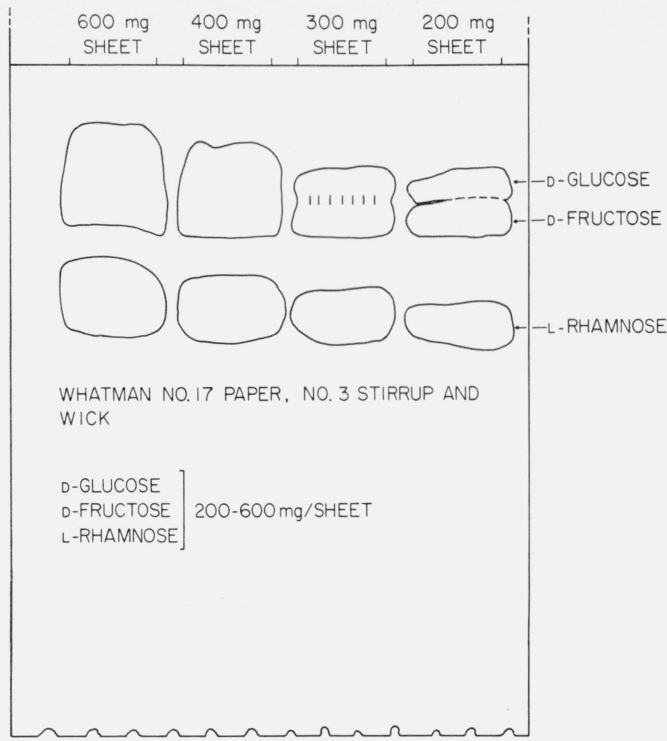

FigURE 6. Illustration of capacity of No. 17 paper.

\section{References}

[1] H. K. Mitchell and F. A. Haskins, Science 110, 278 (1949); W. L. Porter, Anal. Chem. 23, 412 (1951); A. Fischer and M. Behrens, Z. Physiol. Chem. 291, 14 (1952); L. Zechmeister, Science 113, 35 (1951); L. Hagdahl and C. E. Danielson, Nature 174, 1062 (1954); L. B. Rockland, J. Chromatog. 16, 547 (1964).

[2] Instructions for LKB "Chromax" Pressurized Paper Chromatography Column System, LKB-Produkter, Stockholm 12, Sweden.

[3] H. H. Brownell, J. G. Hamilton, and A. A. Casselman, Anal. Chem. 29,550 (1957).

[4] J. N. Balston and B. E. Talbot, A Guide to Filter Paper and Cellulose Powder Chromatography (H. Reeve Angel \& Co., Ltd., London, and W. \& R. Balston Ltd., Maidstone, England, 1952 , p. 21).

[5] J. H. Mueller, Science 112, 405 (1950).

[6] Australian Patent No. 233,713, Imperial Chemical Industries of Australia and New Zealand, Ltd., May 1961.

[7] D. J. MacLaurin, J. K. Crossman, and J. W. Green, ChemistAnalyst 10, No. 4, 118 (1965).

(Paper 71Al-436) 